\title{
Strong and weak lensing united: the cluster mass distribution of the most $\mathrm{X}$-ray luminous cluster RX J1347-1145
}

\author{
Maruša Bradač*t \\ IAEF Bonn, Germany \\ E-mail: marusa@astro.uni-bonn.de
}

\begin{abstract}
Weak gravitational lensing is one of the most powerful tools to study the mass and the mass distribution of galaxy clusters. However, the mass-sheet degeneracy transformation has limited its success. We present a novel method for a cluster mass reconstruction, which combines weak and strong lensing information on common scales and can as a consequence break the mass-sheet degeneracy. We apply the method to ground-based high-quality multi-colour data of RX J1347-1145, the most X-ray luminous cluster to date. The very deep multi-colour data analysis reveals many additional arc candidates in addition to what was previously known for this cluster. The combined strong and weak lensing reconstruction confirms that the cluster is indeed very massive. If the redshift and identification of the multiple-image system as well as the photometric redshift estimates of the source galaxies used for weak lensing are correct, the projected enclosed cluster mass is $M\left(<360 \mathrm{~h}^{-1} \mathrm{kpc}\right)=(1.2 \pm 0.3) \times 10^{15} M_{\odot}$. With higher resolution (e.g. HST imaging data) more reliable multiple imaging information can be obtained and the reconstruction can be improved to accuracies greater than what is currently possible with weak or strong lensing techniques.
\end{abstract}

Baryons in Dark Matter Halos

5-9 October 2004

Novigrad, Croatia

\footnotetext{
${ }^{*}$ Speaker.

${ }^{\dagger}$ In collaboration with T. Erben, P. Schneider, H. Hildebrandt, M. Lombardi, M. Schirmer, J.-M. Miralles, D. Clowe, and S. Schindler.
} 


\section{Introduction}

Clusters of galaxies have long been recognised as excellent laboratories for many cosmological studies. In particular, reliable mass-estimates are important since they provide constraints on the structure formation paradigm. We use a combined strong and weak lensing mass reconstruction to determine the mass and the mass distribution of clusters. We reconstruct the gravitational potential, since it locally determines both the lensing distortion (for weak lensing) as well as deflection (for strong lensing). The method extends the idea from [1]. Its novel feature is that we extend the weak lensing treatment to the critical parts of the cluster and directly include strong lensing information. In addition we use redshift information of the individual background sources and the source(s) being multiply imaged. Without the redshift information, the method would suffer from the masssheet degeneracy transformation of the (scaled) surface mass density $\kappa$ given by $\kappa \rightarrow \kappa^{\prime}=\lambda \kappa+(1-$ $\lambda$ ), where $\lambda$ is an arbitrary constant. At present this degeneracy gives one of the main limitations to measure the cluster masses accurately by using gravitational lensing, however by including the redshift measurements to our method, it can be broken allowing us to accurately measure the cluster mass and mass-distribution. We test this using simulations and apply the method to observational weak and strong lensing data of the cluster RX J1347-1145. In these proceedings we only briefly describe the method and the results, the full work can be found in [2] and [3].

\section{The cluster mass reconstruction method}

We generate weak and strong lensing mock catalogues using the high-resolution N-body simulation by Volker Springel. We construct the catalogues that follow the quality of the data we use in the next section (i.e. number density, redshift distribution, errors on ellipticity measurements) as closely as possible. The two different types of catalogues thus have the spatial and redshift distribution equal to the I and R-band catalogues of RX J1347-1145. We generate a quadruply imaged system at a redshift of $z_{\mathrm{s}}=1.76$ and use as the strong lensing constraints for the reconstruction. The image positions and the original surface mass density $(\kappa)$ map (for the cluster at a redshift of $z_{\mathrm{d}}=0.4$ ) are given in Fig. 1a, and the reconstructed $\kappa$ is given in Fig. $1 \mathrm{~b}$.

From the reconstructed maps we determine the projected mass within a radius of $1.5\left(340 h^{-1} \mathrm{kpc}\right)$ around the cluster centre to be $(1.0 \pm 0.1) \times 10^{15} M_{\odot}$, which is very close to the input value of $M_{\mathrm{s}}\left(<340 h^{-1} \mathrm{kpc}\right)=0.99 \times 10^{15} M_{\odot}$. The error has been estimated from the variance of mass determinations from different mock catalogues. The results show that our method is effectively able to break the mass-sheet degeneracy and is, as a consequence, able to reproducing the cluster mass also at radii different from the Einstein radius of the cluster.

Unfortunately we can not resolve the mass subclumps present in the simulation. This is due to the fact that the number density of background sources is low and the internal smoothing scale (i.e. the average distance between two source galaxies) is large; with a number density of $\sim$ $100 \operatorname{arcmin}^{-2}$ galaxies that can be used for weak lensing the clumps can be easily resolved.

\section{Cluster mass reconstruction of RX J1347-1145}

Due to its record holding, the most X-ray luminous cluster known to date has been the subject of many studies in the X-rays and optical as well as at radio $(\mathrm{mm})$ frequencies. Unfortunately 

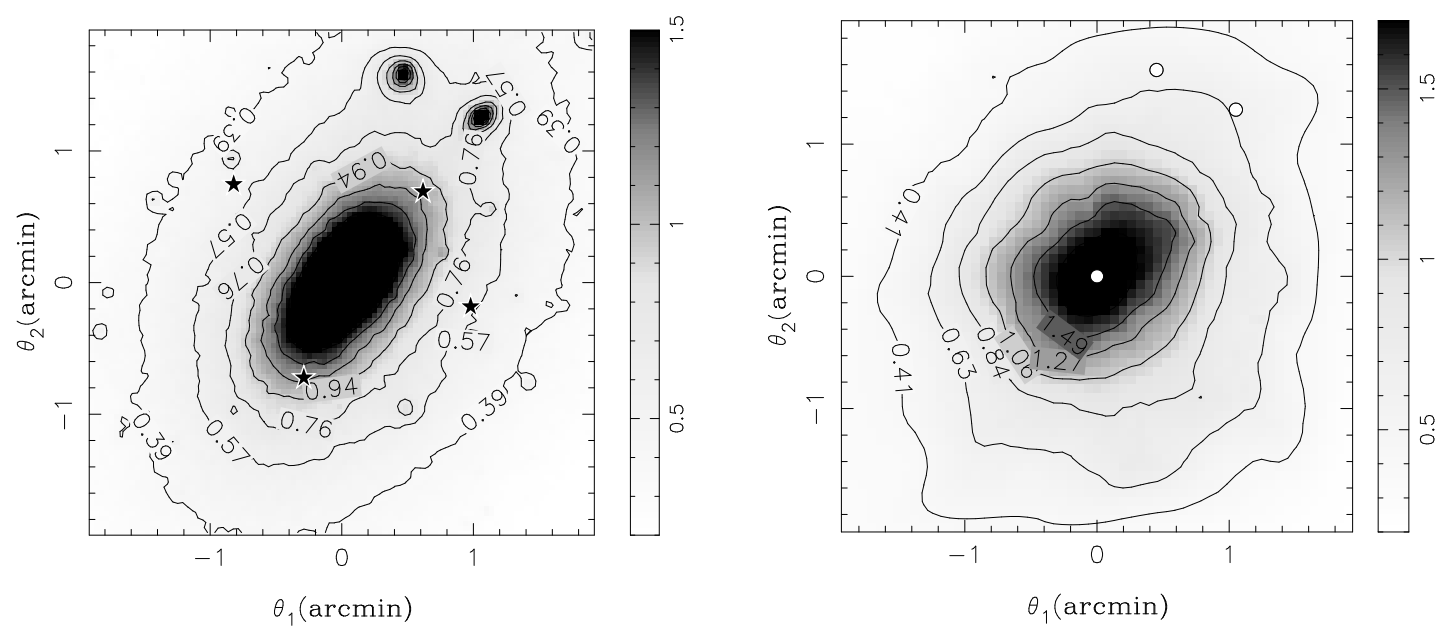

Figure 1: Left: The surface mass density $\kappa$ (for a fiducial source at $z_{\mathrm{s}} \rightarrow \infty$ ) of the simulated cluster used to generate mock catalogues. The stars in the left panel denote the image positions of a four-image system at $z_{\mathrm{s}}=1.76$ we use for the reconstruction. Right: The reconstructed $\kappa$-map (for a fiducial source at $z_{\mathrm{s}} \rightarrow \infty$ ).

studies based on X-ray properties, SZ-effect, velocity dispersion measures, strong and weak lensing have yielded discrepant results for the mass estimate.

Encouraged by the success of the tests of our cluster mass reconstruction method, we apply it to VLT/FORS data on the field of $3.8 \times 3.8 \mathrm{arcmin}^{2}$ in UBVRI bands. Using these we estimate the photometric redshifts of the source galaxies. In addition, we use K-band data from ISAAC to improve the photometric redshift estimates for the strong lensing candidates.

Thus far, five arcs have been reported in the cluster. Unfortunately, these five arcs (see details in [3]) do not belong to the same multiple image system. However two of them do have the same colours and we consider them to belong to the same multiply imaged system. Based on the colours and surface brightness of the images in the field we find a possible candidate counter image which we include in the analysis. Using 6 filters for the redshift determination of the two arcs and 5 filters for the counter image (it is located at the edge of the K-band images and therefore the photometry is not reliable) we find all three images are consistent with being at a redshift of $z_{\mathrm{s}} \simeq 1.76$. In the course of this work we found many new arc candidates, for details see [3].

The cluster RX J1347-1145 is at the redshift $z_{\mathrm{d}}=0.451$ and the weak lensing data consist of $N_{\mathrm{g}}=210$ galaxies from I- and $N_{\mathrm{g}}=145$ galaxies from R-band respectively. The resulting surface mass density maps are given in Fig. 2. We determine the enclosed mass within a radius of 1.5 (corresponding to $360 h^{-1} \mathrm{kpc}$ at the cluster redshift) of the cluster to be $M\left(<360 h^{-1} \mathrm{kpc}\right)=$ $(1.2 \pm 0.3) \times 10^{15} M_{\odot}$.

The resulting mass from the strong and weak lensing mass reconstruction is higher than, but marginally consistent with X-ray measurements for the projected enclosed mass ([4]; M.Gitti, private communication). However since the cluster is most likely in a merger process the south-east quadrant is excluded (the surface brightness profile is determined by averaging data only in the other three quadrants) from the X-ray determination of the cluster mass. Therefore if there is some extra mass present in the excluded quadrant (as suggested by our mass maps), the mass estimate obtained in this way from X-rays could be underestimated. We also extrapolate our mass estimate 
to larger radii (assuming an isothermal profile) and the projected mass is consistent with the previous weak-lensing results. It is however significantly larger than the mass estimate obtained by the velocity dispersion measurement.
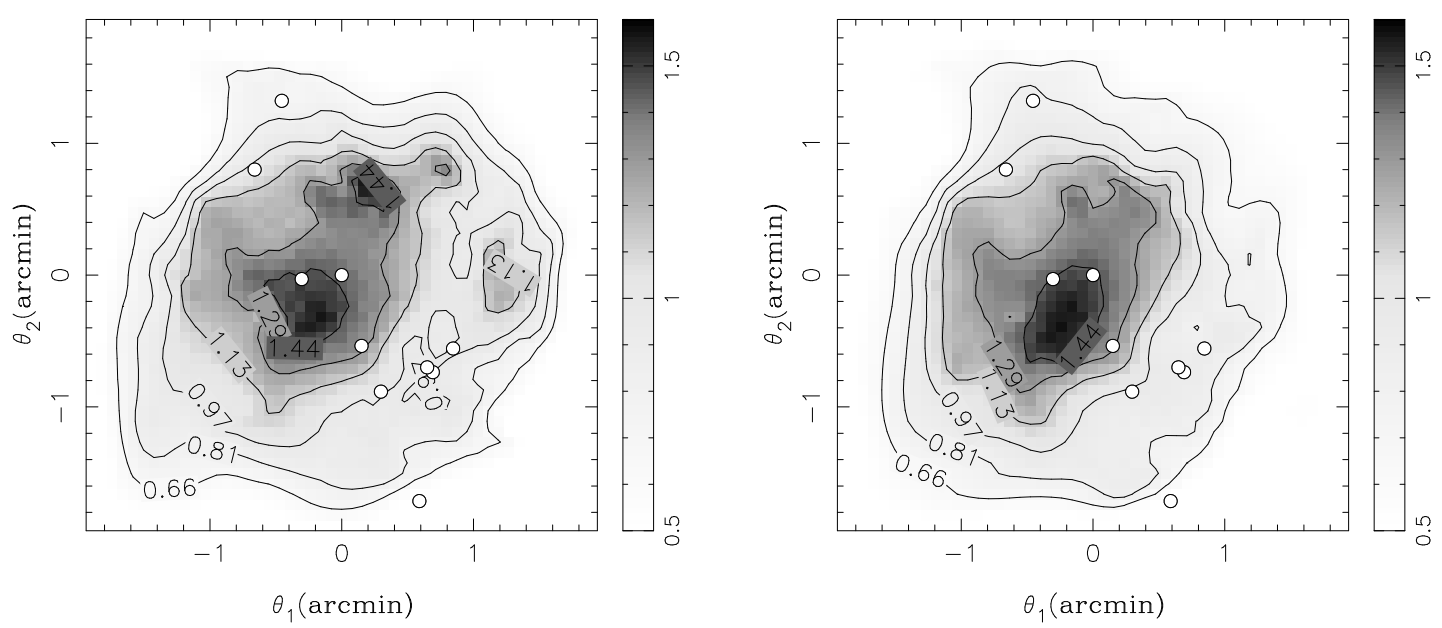

Figure 2: The surface mass density $\kappa$-maps obtained from combined strong and weak lensing reconstruction of the cluster RX J1347-1145. Left panels show the reconstructions using I-band data and for the ones on the right we use the R-band data. The 10 brightest cluster members are plotted as white circles.

\section{Conclusions}

Our main conclusions are the following. (1) A combined strong and weak lensing mass reconstruction shows that the most X-ray luminous cluster is indeed very massive. If the redshift and identification of the multiple-image system, as well as the redshifts used in weak lensing data, are correct we determine the enclosed cluster mass within $360 h^{-1} \mathrm{kpc}$ to $M\left(<360 h^{-1} \mathrm{kpc}\right)=$ $(1.2 \pm 0.3) \times 10^{15} M_{\odot}$. (2) The mass reconstruction shows a south-east mass extension, compatible with the X-ray measurements (see e.g. [4]).

The method has shown great potential for the future. If high quality data is used, the combination of strong and weak lensing offers a unique tool to pin down the masses of galaxy-clusters as well as their profiles and accurately test predictions within the CDM framework.

Acknowledgments: We would like to thank Volker Springel for providing us the N-body simulations. MB acknowledges support from IMPRS (MPIfR Bonn) and GK (University of Bonn/Bochum).

\section{References}

[1] Bartelmann, M., Narayan, R., Seitz, S., and Schneider, P. ApJ 464, L115 June (1996).

[2] Bradač, M., Schneider, P., Lombardi, M., and Erben, T. astro-ph/0410643, November (2004).

[3] Bradač, M., Erben, T., Schneider, P., Hildebrandt, H., Lombardi, M., Schirmer, M., Schindler, S., and Miralles, J.-M. astro-ph/0410642, November (2004).

[4] Gitti, M. and Schindler, S. astro-ph/0409627 September (2004). 